\title{
Caracterização de pacientes com lesão encefálica adquirida submetidos à cirurgia para correção de deformidades nos membros inferiores
}

\author{
Correction of orthopedic deformities deformities Orthopedic in adults \\ with acquired encephalic injury in Adult Acdquired injury
}

Deborah Cristina Garcia de Mattos ${ }^{1}$, Danielle Silva Viegas de Oliveira², Erika Suzigan³, Rita de Cassia Neves ${ }^{4}$, Douglas Martins Braga5

\begin{abstract}
RESUMO
Objetivo: Identificar as características demográficas e clínicas dos pacientes com Lesão Encefálica Adquirida (LEA) submetidos a cirurgias ortopédicas para correção de deformidades em membros inferiores (MMII), e analisar quais procedimentos cirúrgicos foram mais realizados nesses pacientes. Métodos: estudo transversal com revisão de 103 prontuários de pacientes submetidos a cirurgia ortopédica para correção de deformidade em membros inferiores no período de 2012 a 2014. Resultados: Os pacientes com diagnóstico de Traumatismo Cranioencefálico (TCE) e sequela de dupla hemiparesia foram os frequentemente submetidos à cirurgia de correção de partes moles, sendo que o alongamento de tríceps sural a técnica mais realizada. Conclusão: Os pacientes vítimas de TCE foram submetidos a correções cirúrgicas de MMII, seguidos pelos pacientes vítimas de Acidente Vascular Cerebral (AVC), com faixa etária entre 39 e 51 anos, respectivamente, e predominância do sexo masculino, em todos os grupos. As técnicas mais realizadas envolveram a correção de deformidades no pé e tornozelo. Observamos que o tempo transcorrido entre a lesão e o procedimento cirúrgico fora igual ou superior a quatro anos, sendo comum que pacientes chegassem aos centros de reabilitação com deformidades, já instaladas, devido à espera para ingressar em algum serviço.
\end{abstract}

Palavras-chave: Lesões Encefálicas. Adulto. Cirurgia Ortopédica. Deformidades. Membros Inferiores.

1. Fisioterapeuta do setor de fisioterapia aquática da Unidade Central Ibirapuera/ Associação de Assistência à Criança Deficiente (AACD), São Paulo, SP

2. Aperfeiçoanda de Fisioterapia da Unidade Central Ibirapuera Associação de Assistência à Criança Deficiente (AACD), São Paulo, SP

3. Médica Fisiatra da Unidade Central Ibirapuera/Associação de Assistência à Criança Deficiente (AACD), São Paulo, SP

4. Fisioterapeuta do setor de fisioterapia referência na clínica de LEA da Unidade Central Ibirapuera/Associação de Assistência à Criança Deficiente (AACD), São Paulo, SP

5. Fisioterapeuta referência da clínica de poliomielite do setor de fisioterapia aquática da Unidade Central Ibirapuera/ Associação de Assistência à Criança Deficiente (AACD), São Paulo, SP

Trabalho realizado: Associação de Assistência à Criança Deficiente (AACD), São Paulo, SP, Brasil
CORRESPONDÊNCIA Deborah Cristina Garcia De Mattos Associação de Assistência à Criança Deficiente (AACD), Unidade Central Ibirapuera

R. Borges Lagoa, 1505 04038-034 - São Paulo/SP - Brasil deborah.mattos@hotmail.com

Recebido em 20/02/2018 Aprovado em 27/02/2019 


\begin{abstract}
Objective: to identify the demographic and clinical characteristics of patients with acquired brain injury (ABI) submitted to orthopedic surgeries to correct lower limb deformities (LLDF), and to analyze which surgical procedures were more commonly performed in these patients. Methods: Cross-sectional study with a review of 103 medical records of patients undergoing orthopedic surgery for correction of lower limb deformity in the period from 2012 to 2014 . Results: Patients diagnosed with cranioencephalic trauma (CET) and sequelae of double hemiparesis were the ones frequently submitted to soft tissue correction surgery, and the triceps stretching sural was the most accomplished technique. Conclusion: Patients who were victims of CET underwent surgical correction of lower limbs, followed by patients with stroke, with age range between 39 and 51 years, respectively, and male predominance, in all groups. The most accomplished techniques involved the correction of foot and ankle deformities. We observed that the time elapsed between the lesion and the surgical procedure was equal to or greater than four years, and it was common for patients to arrive at rehabilitation centers with deformities, already installed, due to waiting to be admitted in some health service.
\end{abstract}

Keywords: Brain Injuries. Adult. Orthopedic Surgery. Deformities. Lower Extremity.

\section{INTRODUÇÃO}

A Lesão Encefálica Adquirida no Adulto (LEA) envolve as desordens neurológicas que comprometem o cérebro. Dentre as LEA encontram-se o Acidente Vascular Cerebral (AVC), o Traumatismo Cranioencefálico (TCE), as Encefalopatias Anóxicas, os Tumores Encefálicos e as Neuroinfecções, sendo estes três últimos não tão frequentes. ${ }^{1} \mathrm{O}$ AVC é caracterizado por um déficit neurológico súbito causado por uma injúria, não traumática, podendo cursar de forma isquêmica ou hemorrágica. Muito embora dados recentes mostrem que os números de acidentes vasculares cerebrais diminuíram, ainda afeta um grande número de pessoas, muitos destes permanecem com déficits funcionais residuais. ${ }^{2}$ Já no TCE, definido como uma alteração da função cerebral ocasionada por força externa ao encéfalo, ${ }^{3,4}$ lesões traumáticas são a principal causa no mundo e no Brasil, aumentam cada ano. O trauma pode ocorrer em frações de segundo, porém, os prejuízos podem permanecer por longos períodos ou até serem definitivos. Os traumatismos crânio encefálicos afetam principalmente adolescentes e idosos, ${ }^{5,6}$ predominantemente do sexo masculino em todos os aspectos: familiar e socioeconômico. ${ }^{6}$ Segundo a Organização Mundial da Saúde (OMS), podem se tornar a terceira principal causa de deficiência até $2020 .^{5}$

As alterações motoras são comuns após uma lesão ao encéfalo e ocorrem devido ao comprometimento do sistema corticoespinhal, 4,7 levando à diminuição da força, e alteração de tônus. Sabe-se que uma das sequelas decorrentes da lesão do sistema corticoespinhal é a espasticidade, que causa desequilíbrio entre a ação da musculatura agonista e antagonista, afetando a capacidade motora e a funcionalidade dos indivíduos acometidos. ${ }^{8,9} \mathrm{~A}$ espasticidade associada à fraqueza muscular e à diminuição da mobilidade, comum nos indivíduos com LEA que pode levar ao desenvolvimento de deformidade articular no membro inferior do lado comprometido ou de ambos os lados nos pacientes, impactando nas atividades diárias do indivíduo, dificultando, por vezes, o uso de um calçado até mesmo a capacidade de realizar a marcha. ${ }^{10}$

O prejuízo na capacidade de realizar a marcha é uma das principais consequências do AVC e do TCE, e recuperar a capacidade de deambulação é uma das metas principais do processo de reabilitação. ${ }^{9,11,12}$ Formas de minimizar o impacto negativo da LEA na marcha incluem o uso de órteses de posicionamento (como a órtese suropodálica), a realização de bloqueios neuroquímicos, cinesioterapia, cirurgias neurolíticas seletivas e também cirurgias ortopédicas, quando a deformidade já está instalada. ${ }^{10,13,14}$

Já é bem documentado o sucesso das cirurgias de partes moles para correção de deformidades de tornozelos e pés. ${ }^{13,15,16}$ no entanto, são escassos na literatura trabalhos que apontem as características dos pacientes submetidos a essas cirurgias, bem como quais as deformidades são mais encontradas nessa população, além das 
deformidades de pé e tornozelo. Sendo assim, o objetivo deste trabalho foi identificar as características demográficas e clínicas dos pacientes com LEA submetidos a cirurgias ortopédicas para correção de deformidades em membros inferiores (MMII), e verificar quais os procedimentos cirúrgicos são mais realizados nesses pacientes.

\section{MATERIAIS E MÉTODOS}

Trata-se de um estudo transversal, com revisão de prontuários, desenvolvido na Associação de Assistência à Criança Deficiente (AACD), unidade Ibirapuera, São Paulo. As questões éticas da pesquisa foram aprovadas, conforme os parâmetros da resolução $466 / 12$, sob o parecer CAEE 5308.2716.0.0000.0085.

Participaram do estudo pacientes com diagnóstico de LEA decorrentes de AVC, TCE, Encefalopatia Anóxica e Neuroinfecções, com idade superior a 16 anos e 11 meses, ambos os sexos, submetidos a cirurgia ortopédica para correção de deformidades nos MMIIs, no período de janeiro de 2012 a dezembro de 2014. Foram excluídos os pacientes que apresentaram outras doenças neurológicas concomitantes, que não fizeram acompanhamento pós-operatório na instituição ou apresentaram dados incompletos no prontuário.

A coleta de dados foi feita através da análise do prontuário dos pacientes, tais como, dados pessoais (nome, data de nascimento, sexo e idade), dados clínicos (causa e data da LEA, sequela motora) e da cirurgia realizada (data da cirurgia, técnica e objetivo da correção cirúrgica, tecido abordado cirurgicamente).

Em relação ao tecido abordado, foram consideradas cirurgias de partes moles (alongamento e/ou transferência tendínea); partes ósseas (ressecção, osteotomia óssea ou artrodese articular); e partes moles e ósseas realizadas no mesmo procedimento, ou seja, o mesmo paciente poderia ter sido submetido a mais de uma correção na mesma cirurgia, e todas as correções foram computadas. As informações foram inseridas para o programa Microsoft Excel 2015 e analisadas de forma descritiva dos dados.

\section{RESULTADOS}

Foram selecionados 106 pacientes, porém, três foram excluídos por apresentarem dados incompletos no prontuário. Assim, foram analisados 103 pacientes submetidos a intervenção cirúrgica no período de janeiro de 2012 a dezembro de 2014.

O diagnóstico, dados demográficos e o tempo transcorrido entre a lesão e a data da cirurgia ortopédica são apresentados na Tabela 1. Os pacientes com diagnóstico de TCE foram os que mais se submeteram à correção cirúrgica, sendo a dupla hemiparesia a sequela mais frequente nesse grupo. O sexo masculino foi predominante em todos os grupos, e a idade variou conforme a etiologia da lesão. Nos grupos de Encefalopatia Anóxica e TCE, a média de idade foi menor que no grupo de AVC sendo, 34 e 39 anos, respectivamente, enquanto no grupo AVC a média foi maior (51 anos).

\section{Tabela 1}

Diagnóstico, dados demográficos e média de tempo entre a lesão X correção cirúrgica.

\begin{tabular}{|c|c|c|c|c|c|c|c|c|c|c|}
\hline \multicolumn{3}{|c|}{ Diagnóstico } & \multicolumn{4}{|c|}{ Sexo } & \multicolumn{2}{|c|}{ Idade } & \multicolumn{2}{|c|}{$\begin{array}{c}\text { Tempo de lesão } \\
\text { x } \\
\text { Correção cirúrgica }\end{array}$} \\
\hline & & & & $M$ & & $\mathrm{~F}$ & Média & DP & Média & DP \\
\hline & $\mathrm{N}$ & $\%$ & $\mathrm{~N}$ & $\%$ & $\mathrm{n}$ & $\%$ & & & & \\
\hline TCE & 57 & $55,3 \%$ & 45 & $78,9 \%$ & 12 & $21,1 \%$ & 39,7 & $\pm 12,1$ & 6,8 & $\pm 4,9$ \\
\hline AVC & 36 & $35,0 \%$ & 22 & $61,1 \%$ & 14 & $38,9 \%$ & 51,6 & $\pm 12,0$ & 8,1 & $\pm 6,8$ \\
\hline $\begin{array}{l}\text { Encefalopatia } \\
\text { Anóxica }\end{array}$ & 7 & $6,8 \%$ & 5 & $71,4 \%$ & 2 & $28,6 \%$ & 34,0 & $\pm 13,2$ & 4,1 & $\pm 1,1$ \\
\hline Neuroinfecção & 3 & $2,9 \%$ & 2 & $66,7 \%$ & 1 & $33,3 \%$ & 40,7 & $\pm 8,3$ & 10,3 & $\pm 6,7$ \\
\hline Total & 103 & $100 \%$ & 74 & $71,8 \%$ & 29 & $28,2 \%$ & 41,5 & $\pm 11,4$ & 7,32 & 4,87 \\
\hline
\end{tabular}

*TCE: Traumatismo Cranioencefálico

${ }^{* *}$ AVC: Acidente Vascular Cerebral 
Notamos que, os pacientes com sequela de Neuroinfecção tiveram maior intervalo entre o diagnóstico e a correção cirúrgica, enquanto esse intervalo foi menor para os pacientes com sequela de Encefalopatia Anóxica. Isso pode ser considerado devido a importante alteração do tônus e manifestação difusa comum na Encefalopatia Anóxica.
O Gráfico 1 demonstra as correções cirúrgicas realizadas nos pacientes com sequela de TCE, AVC, Encefalopatia Anóxica e Neuroinfecções, respectivamente. $\mathrm{O}$ alongamento do Tríceps Sural foi a correção mais realizada nos pacientes com sequela de TCE e AVC, e a segunda mais realizada nos demais grupos.

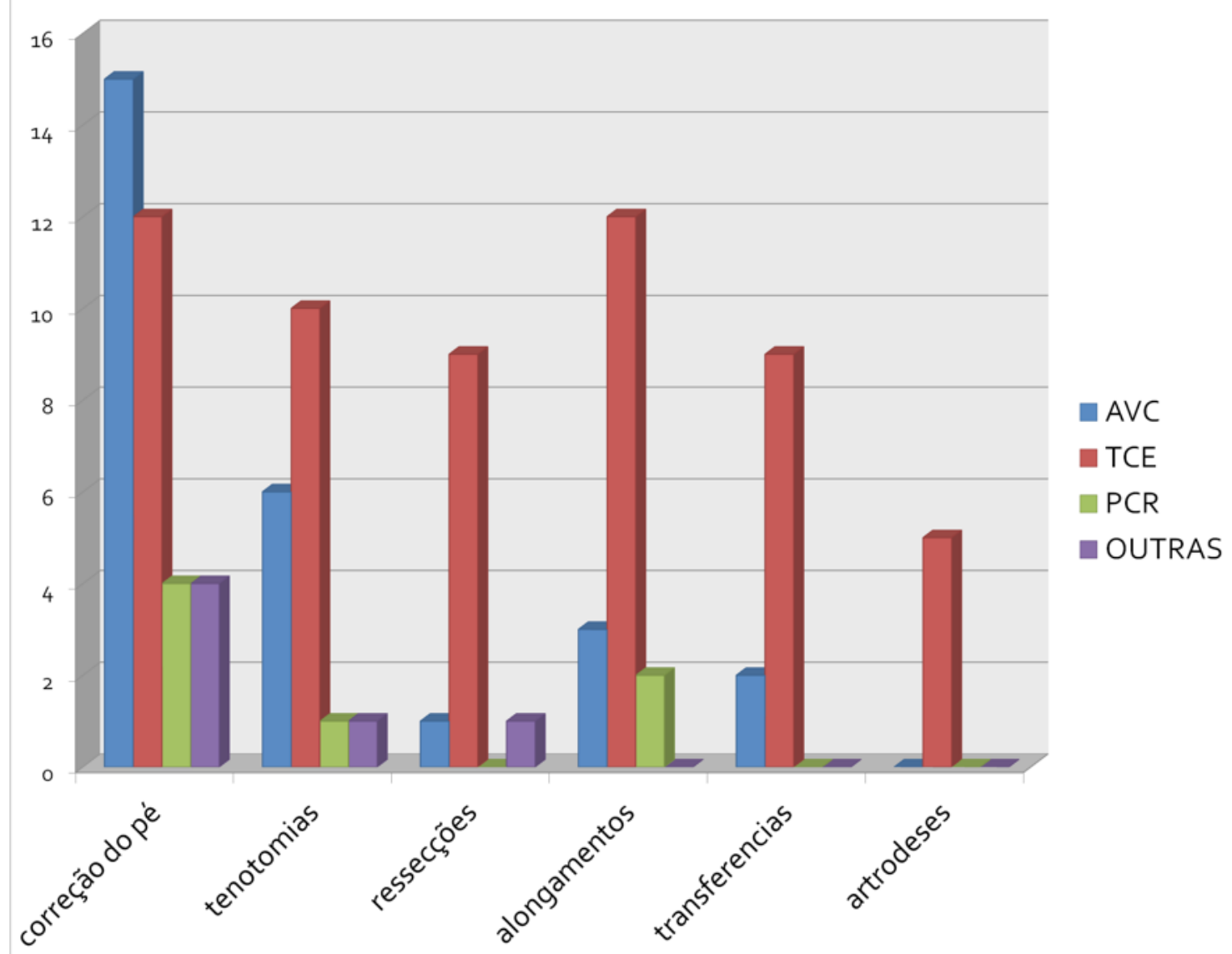

Grafico 1: Correções cirúrgicas realizadas nos pacientes com sequela de AVC, TCE, PCR e outras.

O Alongamento do flexor longo e curto dos dedos e hálux foram à segunda cirurgia mais frequente em todos os grupos estudados. Em relação ao tecido mais abordado cirurgicamente (Gráfico 2), houve predominância de correção em partes moles, seguido por partes moles e ósseas na mesma cirurgia; as cirurgias com correção somente de partes ósseas, foram as menos frequentes e relacionadas à ressecção de Ossificação Heterotópica que podem ocorrer com maior frequência em pacientes com sequela de TCE. 


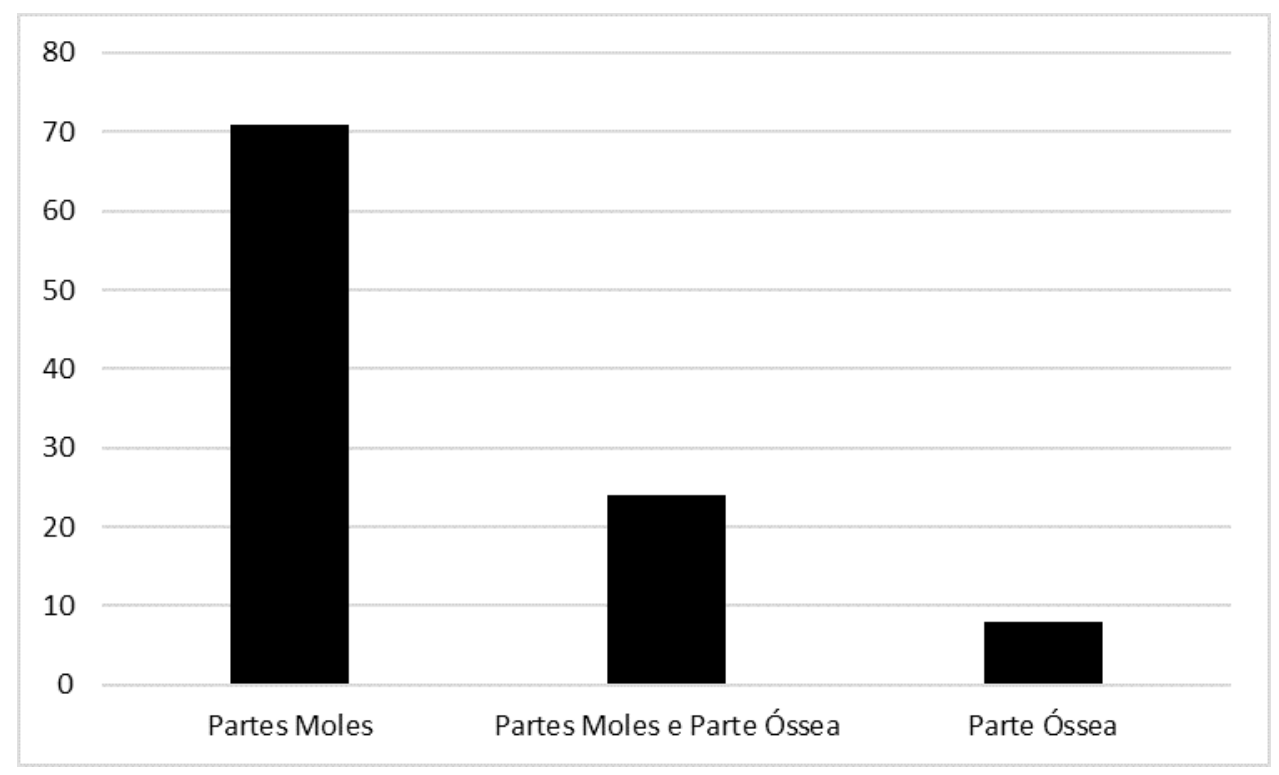

Gráfico 2: Tecidos mais abordados nas correções cirúrgicas dos pacientes com LEA.

\section{DISCUSSÃO}

O objetivo deste estudo visa identificar as características demográficas e clínicas dos pacientes submetidos a cirurgias ortopédicas para correção de deformidades em MMII e verificar quais procedimentos cirúrgicos são mais realizados nesses pacientes.

Embora o AVC seja a principal causa de LEA em adultos, ${ }^{17}$ em nossa amostra, os pacientes com TCE foram os mais submetidos a correções cirúrgicas. Não há na literatura relatos de quais grupos de indivíduos são mais submetidos a essas cirurgias. No entanto, neste estudo, as vítimas de TCE apresentaram uma sequela motora mais difusa, o que supõe uma maior exposição ao aparecimento de contraturas e deformidades, e, portanto, incapacidade funcional importante, que pode justificar o maior número de pacientes vítimas de TCE submetidos a correções cirúrgicas. Cerca de $70 \%$ dos sobreviventes de TCE podem readquirir a marcha dentro dos primeiros seis meses pós-trauma. ${ }^{9,12}$ Nesses casos, as cirurgias ortopédicas são uma forma de facilitar a deambulação, gerando menor número de compensações e muitas vezes dispensando o uso de órteses. ${ }^{9} \mathrm{O}$ mesmo pode acontecer para $60-80 \%$ dos pacientes com sequelas de AVC, que podem recuperar a marcha nos primeiros seis meses pós-lesão. ${ }^{9,18,19}$
Em ambos os grupos, as articulações do tornozelo e pés foram as mais abordadas cirurgicamente. Embora diferentes tipos de deformidades adquiridas de MMII sejam documentadas, o pé equino varo é a mais frequente nesse grupo de pacientes ${ }^{9,20} \mathrm{O}$ aumento do tônus e a atividade involuntária dos flexores plantares e dos artelhos, em conjunto com a fraqueza dos músculos flexores dorsais e eversores podem explicar a instalação do pé equino varo. 9,21,22 Tal posicionamento do segmento distal do membro inferior dificulta a postura ortostática, a marcha traz desconforto para os pacientes, dificultando até mesmo o uso de calçados.9,22 Segundo Renzenbrink et al. (2012), "a correção cirúrgica do pé equino varo é segura e pode ser eficaz para posicionar o pé e melhorar a capacidade de marcha", que é um dos principais objetivos desses pacientes durante a reabilitação. ${ }^{23}$

Além da correção de deformidades do pé e tornozelo, as correções na articulação do joeIho, envolvendo a liberação dos isquiotibiais ou do reto femoral também foram frequentes em nossa amostra, indicando que o envolvimento dessa musculatura é frequente nesse grupo e pode ser um fator incapacitante para a manutenção da postura ortostática no caso da flexão de joelhos ou a fase de balanço da marcha. No caso da excessiva ativação do reto femoral destes indivídu- 
os, foi observado um número maior de correção da flexão do joelho na população deste estudo.

Em relação ao tempo transcorrido entre a lesão e a abordagem cirúrgica em nossa amostra, a maior parte dos pacientes levou quatro anos ou mais para ser submetido ao procedimento cirúrgico. Não há, na literatura, um consenso sobre quando a abordagem cirúrgica deve ser realizada.

Em geral, os pesquisadores optam por aguardar a fase de recuperação neurológica que pode variar de seis a nove meses após o AVC e até 12 a 18 meses após o TCE. ${ }^{9}$ A abordagem cirúrgica para correção do pé equino varo e também de outras deformidades de MMII é geralmente considerada após a ineficiência de outros tratamentos. ${ }^{9}$ No entanto, em uma abordagem precoce, poderia evitar a instalação de estratégias compensatórias na archa, além de diminuir gastos com outros métodos de tratamento não permanentes. ${ }^{9}$ Renzenbrink et al. (2012) observaram que a recorrência de pé equino varo após a cirurgia é rara, e que esta pode ser uma opção duradoura de tratamento, além de proporcionar outros benefícios ao paciente, como a possibilidade de caminhar descalço ou sem o uso de órteses. ${ }^{9,} 23$

Outro fator a ser considerado é o tempo que o indivíduo leva para chegar ao Centro de Reabilitação, já que em alguns serviços de saúde, esse processo pode levar alguns anos. É comum o paciente ser recebido no Centro de Reabilitação já com sequelas crônicas, anos após a lesão encefálica e após ter passado por outros serviços onde a correção cirúrgica da deformidade não é uma realidade. Isso corrobora para o grande número de pacientes submetidos à cirurgia com lesões antigas no nosso estudo, pois, associamos com os ortopedistas a este procedimento, o que acaba sendo facilmente indicado.

Ainda, são comuns nesses casos a ocorrência de deformidades já instaladas, onde a abordagem apenas de partes moles não pode mais ser considerada, necessitando também de correção óssea.

Em decorrência da falta de informações sobre as condições pré-operatórias desses pacientes, tais como amplitude de movimento, força e tônus muscular, não nos permitiu traçar perfil mais detalhado, assim como o seguimento no pós-operatório para verificar o grau de satisfação com o resultado da cirurgia e o status funcional pré e pós-cirurgia, o que seria de grande e melhor diagnóstico. Sugerimos que, em trabalhos futuros, essas características sejam abordadas.

\section{CONCLUSÃO}

No presente estudo os pacientes vítimas de TCE foram mais submetidos a correções cirúrgicas de MMII, seguidos pelos pacientes vítimas de AVC, a faixa etária média de idade de 39 e 51 anos, respectivamente, com predominância do sexo masculino em todos os grupos.

A dupla hemiparesia foi a sequela mais frequente nas vítimas de TCE, Anóxica e Neuroinfecções, enquanto a hemiparesia foi a mais comum nas vítimas de AVC conforme encontrado na literatura.

As deformidades mais tratadas foram a correção de partes moles que envolvem os alongamentos musculares, as transferências tendíneas, envolvendo correções do pé e tornozelo, sendo o Alongamento do Tríceps Sural a mais comum nos grupos TCE e AVC.

Com relação às articulações, as ossificações foram comumente encontradas e tratadas na região dos quadris e nos pacientes com comprometimento mais difuso, como é o caso do paciente com sequela de TCE, encefalopatia anóxica, quando comparado com pacientes com sequela de AVC e neuroinfecções.

Por fim, concluímos que é primordial que os pacientes sejam munidos com todo tipo de informação no pré e pós-operatório para cada diagnóstico do melhor tratamento a se seguir.

\section{Conflito de interesses}

Os autores declaram inexistência de conflito de interesses na realização deste trabalho.

\section{REFERÊNCIAS}

1. Duncan PW, Propst M, Nelson SG. Reliability of the Fugl-Meyer assessment of sensorimotor recovery following cerebrovascular accident. Phys Ther. 1983;63:1606-10. 
2. Winstein CJ, Stein J, Arena R, Bates B, Cherney LR, Cramer SC, et al. Guidelines for Adult Stroke Rehabilitation and Recovery: A Guideline for Healthcare Professionals From the American Heart Association/American Stroke Association. Stroke. 2016;47:e98-e169.

3. Abou-Abbass $\mathrm{H}$, Bahmad H, Ghandour H, Fares J, Wazzi-Mkahal R, Yacoub B, et al. Epidemiology and clinical characteristics of traumatic brain injury in Lebanon: A systematic review. Medicine (Baltimore). 2016;95:e5342.

4. Menon DK, Schwab K, Wright DW, Maas AI. Position Statement: Definition of Traumatic Brain Injury. Arch Phys Med Rehabil. 2010; 91:1637-40.

5. Shivaji T, Lee A, Dougall N, McMillan T, Stark C. The epidemiology of hospital treated traumatic brain injury in Scotland. BMC Neurol. 2014;14:2.

6. Tran TM, Fuller AT, Kiryabwire J, Mukasa J, Muhumuza M, Ssenyojo $H$, Haglund MM. Distribution and characteristics of severe traumatic brain injury at Mulago National Referral Hospital in Uganda. World Neurosurg. 2015;83:269-77.

7. Keenan MA. The management of spastic equinovarus deformity following stroke and head injury. Foot Ankle Clin. 2011;16:499-514.

8. Sathian K, Buxbaum LJ, Cohen LG, Krakauer JW, Lang $C E$, Corbetta $M$, et al. Neurological principles and rehabilitation of action disorders: common clinical deficits. Neurorehabil Neural Repair. 2011;25(5 Suppl):21S-32S.

9. Dobkin $\mathrm{BH}$. Clinical practice. Rehabilitation after stroke. N Engl J Med. 2005;352:1677-84.

10. Katz DI, White DK, Alexander MP, Klein RB. Recovery of ambulation after traumatic brain injury. Arch Phys Med Rehabil. 2004;85:865-9.

11. Giannotti E, Merlo A, Zerbinati P, Longhi M, Prati P, Masiero $S$, et al. Early rehabilitation treatment combined with equinovarus foot deformity surgical correction in stroke patients: safety and changes in gait parameters. Eur J Phys Rehabil Med. 2016;52:296-303.

12. Kamath AF, Pandya MD, Namdari S, Hosalkar H, Keenan MA. Surgical technique for the correction of adult spastic equinovarus foot. Tech Foot Ankle Surg. 2009;8:160-7.
13. Keenan MA, Creighton J, Garland DE, Moore T. Surgical correction of spastic equinovarus deformity in the adult head trauma patient. Foot Ankle. 1984;5:35-41.

14. Roper BA, Williams A, King JB. The surgical treatment of equinovarus deformity in adults with spasticity. J Bone Joint Surg Br. 1978;60-B:533-5.

15. Waters RL, Frazier J, Garland DE, Jordan C, Perry J. Electromyographic gait analysis before and after operative treatment for hemiplegic equinus and equinovarus deformity. J Bone Joint Surg Am. 1982;64:284-8.

16. Bensenor IM, Goulart AC, Szwarcwald CL, Vieira ML, Malta DC, Lotufo PA. Prevalence of stroke and associated disability in Brazil: National Health Survey--2013. Arq Neuropsiquiatr. 2015;73:746-50.

17. Sá BP, Grave MTQ, Périco E. Perfil de pacientes internados por Acidente Vascular Cerebral em hospital do Vale do Taquari/RS. Rev Neurociênc. 2014;22:381-87.

18. Oliveira CO, Ikuta N, Regner A. Biomarcadores prognósticos no traumatismo crânio-encefálico grave. Rev bras ter intensiva. 2008;20:411-21.

19. Jørgensen HS, Nakayama H, Raaschou HO, Olsen TS. Recovery of walking function in stroke patients: the Copenhagen Stroke Study. Arch Phys Med Rehabil. 1995;76:27-32.

20. Kollen B, Van de Port I, Lindeman E, Twisk J, Kwakkel G. Predicting improvement in gait after stroke: a longitudinal prospective study. Stroke. 2005;36:2676-80.

21. Veerbeek JM, Van Wegen EE, Harmeling-Van der Wel BC, Kwakkel G, Investigators E. Is accurate prediction of gait in nonambulatory stroke patients possible within 72 hours poststroke? The EPOS study. Neurorehabil Neural Repair. 2011;25:268-74.

22. Forese L, Wootten M, Kadaba MP, McCann PD. Surgical management of equinovarus deformity in the adult with head injury. Orthop Rev. 1993;22:1001-10.

23. Renzenbrink GJ, Buurke JH, Nene AV, Geurts AC, Kwakkel G, Rietman JS. Improving walking capacity by surgical correction of equinovarus foot deformity in adult patients with stroke or traumatic brain injury: a systematic review. J Rehabil Med. 2012;44:614-23. 